\title{
Internalization of adhesion junction proteins and their association with recycling endosome marker proteins in rat seminiferous epithelium
}

\author{
J'Nelle S Young, Yoshimi Takai ${ }^{1}$, Katarina L Kojic and A Wayne Vogl \\ Department of Cellular and Physiological Sciences, Faculty of Medicine, Life Sciences Centre, The University of \\ British Columbia, 2350 Health Sciences Mall, Vancouver, British Columbia, Canada V6T $1 Z 3$ and ${ }^{1}$ Division of \\ Molecular Biology and Biochemistry, Graduate School of Medicine, Kobe University, Kusunoki-cho, Chuo-ku, \\ Kobe 650-0017, Japan \\ Correspondence should be addressed to A W Vogl; Email: vogl@mail.ubc.ca
}

\begin{abstract}
Tubulobulbar complexes (TBCs) are elaborate cytoskeleton-related structures that are formed in association with intercellular junctions in the seminiferous epithelium. They consist of a cylindrical double-membrane core composed of the plasma membranes of the two attached cells, cuffed by a dendritic network of actin filaments. TBCs are proposed to be subcellular machines that internalize intercellular junctions during the extensive junction remodeling that occurs during spermatogenesis. At the apical sites of attachment between Sertoli cells and spermatids, junction disassembly is part of the sperm release mechanism. In this study, we used immunological probes to explore junction internalization and recycling at apical TBCs in the rat seminiferous epithelium. We demonstrate that $\beta 1$-integrin and nectin 2 were concentrated at the ends of TBCs and for the first time show that the early endosome marker RAB5A was also distinctly localized at the ends of TBCs that appear to be the 'bulbar' regions of the complexes. Significantly, we also demonstrate that the 'long-loop' recycling endosome marker RAB11 A was co-distributed with nectin 2 at junctions with early spermatids deeper in the epithelium. Our results are consistent with the hypothesis that TBCs associated with late spermatids internalize adhesion junctions and also indicate that some of the internalized junction proteins may be recycled to form junctions with the next generation of spermatids. Reproduction (2012) 143 347-357
\end{abstract}

\section{Introduction}

The turnover of massive intercellular junctions in the seminiferous epithelium of the mammalian testis occurs at specific times during spermatogenesis and is associated with two processes that are fundamental to male fertility: 1) the movement of the next generation of spermatogenic cells through the blood-testis barrier and 2) the release of morphologically mature spermatids from the apex of the epithelium. At the basal sites of attachment between neighboring Sertoli cells, the junctions form a complex of tight, gap, 'desmosome-like' junctions, and unique adhesion junctions termed ectoplasmic specializations (ESs; Vogl et al. 2008). Tight junctions within this junction complex form the 'blood-testis barrier' (Dym \& Fawcett 1970). At the apical sites of attachment between Sertoli cells and late spermatids, the junctions consist almost entirely of ESs.

Generally in epithelia, adhesion and tight junction turnover involves the disengagement of junction molecules in one cell from those in the other cell and the internalization of the molecules by conventional clathrin- or caveolin-dependent endocytosis (Le et al. 1999, Akhtar \& Hotchin 2001, Ivanov et al. 2004, Izumi et al. 2004, Shen \& Turner 2005, Troyanovsky et al. 2006, Baum \& Georgiou 2011) or by clathrin- and caveolin-/raft-independent mechanisms such as macropinocytosis (Utech et al. 2005), depending on cell context and regulation (Paterson et al. 2003). The molecules then enter recycling or degradation pathways. Integrins at focal adhesions are also internalized by clathrin-dependent endocytosis (Chao \& Kunz 2009). Intact gap junctions, consisting of connexions in the plasma membranes of the two attached cells, are typically internalized as double-membrane vesicles by one of the cells and are targeted to lysosomes or are recycled (Gaietta et al. 2002, Berthoud et al. 2004), and are clathrin dependent (Piehl et al. 2007). In the seminiferous epithelium, something very different appears to happen. Here, specific subcellular structures called 'tubulobulbar complexes' (TBCs) are formed at the junctions during turnover and are hypothesized to 
internalize 'intact' intercellular junctions into Sertoli cells (Russell 1979b, Guttman et al. 2004).

TBCs consist of elongated tubular extensions of one cell (either a spermatid or a Sertoli cell) that protrude into corresponding invaginations of the adjacent Sertoli cell (Russell \& Clermont 1976, Russell 1979b, Guttman et al. 2004). Within each complex, the double-membrane tubular core is cuffed by a dendritic actin network (Young et al. 2009a). In addition, a clathrin-coated pit occurs at the end of each complex (Young et al. 2009a). As each TBC matures, a swelling or 'bulbar' region forms in the distal third of the structure. This bulbar region separates, or buds, from the complex and is thereby internalized by Sertoli cells (Russell \& Clermont 1976, Russell 1979c). TBCs are dramatically evident at the apical sites of attachment between Sertoli cells and spermatids before the actual disengagement of spermatids from the epithelium and also occur at basal junctions between neighboring Sertoli cells during turnover of the bloodtestis barrier. The process of internalization proposed for TBCs somewhat resembles 'clathrin-mediated bulk endocytosis', a process recently proposed on the basis of results from a cell-free model system (Wu et al. 2010).

If TBCs are subcellular machines that internalize intercellular junctions, then the structures should contain junction molecules and be associated with endosomal markers. At the sites of adhesion between
Sertoli cells and spermatids in the mouse, we had previously shown that the adhesion molecules nectin 2 (PVRL2; in the Sertoli cell plasma membrane) and nectin 3 (PVRL3; in the spermatid plasma membrane) are localized to vesicles associated with the distal ends of TBCs (Guttman et al. 2004), and these vesicles are associated with the early endosomal marker 'early endosome antigen 1' (EEA1; Young et al. 2009b). In the rat, a6-integrin (ITGA6), another adhesion molecule present at adhesion sites (in the Sertoli cell plasma membrane), has also been shown at the ends of TBCs (Young et al. 2009b); however, other adhesion proteins and early endosomal markers have not yet been localized to these sites in the rat. In this study, we demonstrate that $\beta 1$-integrin (ITGB1) and nectin 2 are concentrated at the ends of TBCs in the rat, and for the first time in any species demonstrate that these regions are also associated with the early endosomal marker Rab5 (RAB5A). Significantly, we demonstrate that Rab11 (RAB11A), a marker for the 'long-loop' recycling pathway (Caswell \& Norman 2006), occurs at vesicular-like structures where adhesion junctions are newly forming adjacent to early spermatids and that the marker is co-distributed with PVRL2-positive vesicles at the same sites. 'Short-loop' and 'long-loop' endocytic pathways refer to two spatially and temporally distinct mechanisms controlled by Rab4 (RAB4A) and Rab11A

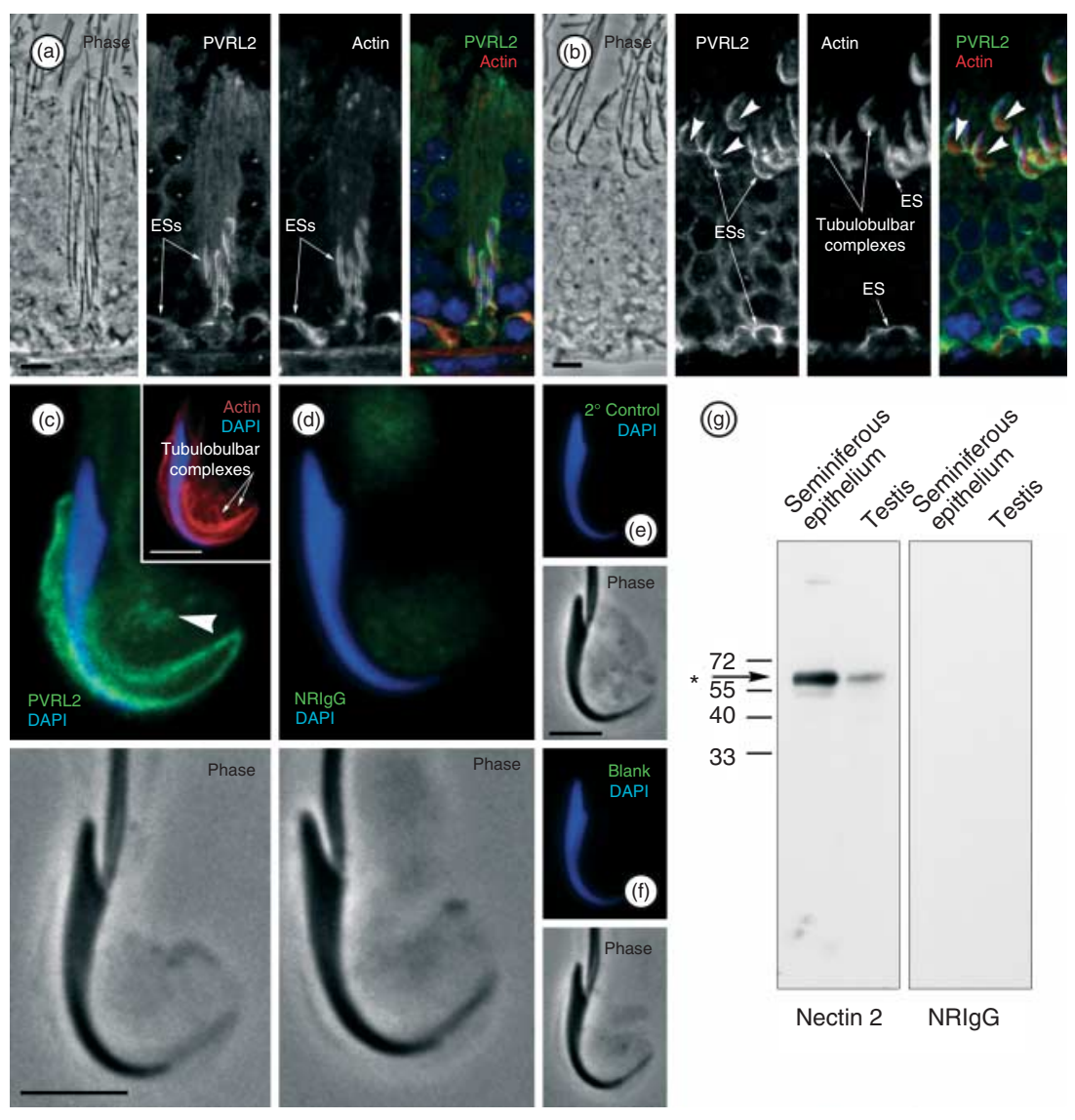

Figure 1 Grouped phase and fluorescence images showing the distribution of PVRL2 (nectin 2) and actin in rat seminiferous epithelium. The distribution of PVRL2 and actin at stages (a) V and (b) VII, respectively, of the seminiferous epithelium. At both stages, nectin 2 is concentrated at ESs, indicated by positive phalloidin staining, both at basal junction complexes and at apical sites of attachment to spermatids. (b) At stage VII, nectin 2 is also concentrated at focal sites (arrowheads) in apical lobes that envelop late spermatid heads. (c) At higher magnification, each of these focal sites appears as a cluster of small vesicular-like structures (arrowhead) at the ends of TBCs identified by actin labeling (inset in (c)). Similar clusters are absent in material treated with (d) normal rabbit $\lg G$ at the same concentration as primary antibody, (e) with buffer alone instead of primary antibody, and (f) with buffer instead of both the primary and the secondary antibodies. (g) In western blots of rat testis and seminiferous epithelium, the nectin 2 antibody reacts strongly with a band (asterisk) between 60 and $65 \mathrm{kDa}$ that is not present in blots treated with normal rabbit $\operatorname{lgG}(\mathrm{NRIgG})$ at the same concentration as primary antibody. Bars $=(\mathrm{a}, \mathrm{b}) 10 \mu \mathrm{m}$ and (c-f) $5 \mu \mathrm{m}$. 

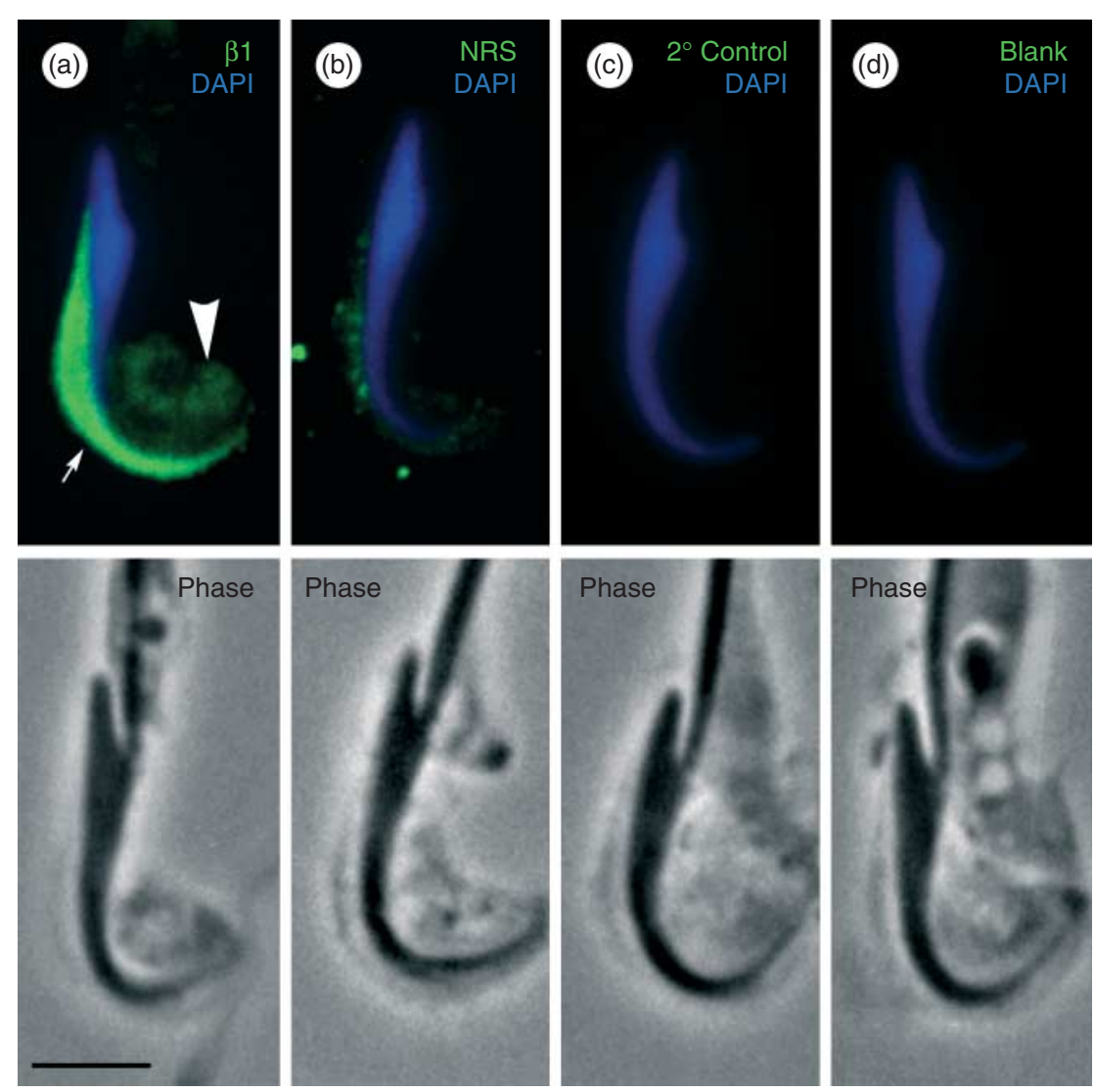

Figure 2 Paired phase and fluorescence micrographs of late spermatids and associated Sertoli cell regions of the rat processed by the antigen retrieval protocol as indicated in the text and labeled with $\beta 1$-integrin antibodies. The antibody clearly labels material in apical Sertoli cell lobules in regions known to contain the ends of TBCs (arrowhead in (a)). Regions known to contain ESs label intensely with the antibodies (small arrow in (a)). Similar patterns of staining are not present in material treated with normal rabbit serum (NRS) at the same concentration as the primary antibody (b), with buffer instead of primary antibody (c), and with buffer instead of both primary and secondary antibodies (d). Bar $=5 \mu \mathrm{m}$. NRS, normal rabbit serum; DAPI, 6-diamidino-2-phenylindole; TBC, tubulobulbar complex; ES, ectoplasmic specialization.
GTPases respectively. The co-distribution of nectin 2 and RAB11A is consistent with the hypothesis that TBCs at the apical sites of attachment internalize adhesion junctions in the rat. Furthermore, our results indicate for the first time that at least some of the junction proteins may be recycled to newly forming junctions with the next generation of spermatids.

\section{Results}

\section{PVRL2 and the $\beta 1$-integrin subunit are concentrated at the ends of apical TBCs}

Antibodies to both PVRL2 (Fig. 1) and $\beta 1$-integrin (Fig. 2) labeled Sertoli cell regions adjacent to the heads of late spermatids consistent with the position of ESs and material in Sertoli cell apical lobules at the ends of TBCs.

The probe for nectin 2 clearly labeled basal junction complexes between Sertoli cells and regions of Sertoli cell attachment to spermatids. This was very evident at stage $V$ of the seminiferous epithelium (Fig. 1a) where spermatids were entrenched deep within apical Sertoli cell crypts. This staining pattern overlapped with the staining of filamentous actin known to be concentrated in ESs at adhesion junctions. At stage VII, when mature spermatids were at the apex of the epithelium, nectin 2 staining was also co-distributed with actin staining at apical and basal ESs (Fig. 1b). In addition, focal nectin 2 staining occurred in the central regions of Sertoli cell apical lobules surrounding late spermatids (arrowheads in Fig. 1b), regions known to contain TBCs. At higher magnification of epithelial fragments labeled for actin, this staining was concentrated at the ends of TBCs (Fig. 1C). Similar staining patterns at ESs and at the ends of TBCs were absent when the primary antibody was replaced with the same concentration of normal rabbit immunoglobulin G (IgG) (Fig. 1d), when the primary antibody was replaced with buffer alone (Fig. 1e), and when both primary and secondary antibodies were replaced with buffer alone (Fig. 1f). In addition, the antibody reacted with a single band of the appropriate molecular weight for nectin 2 (60-65 kDa) on immunoblots of whole testis and isolated seminiferous epithelium not present on control blots treated with normal rabbit IgG instead of the primary antibody (Fig. 1g).

In epithelial fragments processed for antigen retrieval, the antibody to the $\beta 1$-integrin subunit reacted with two regions associated with late spermatids: regions known to contain ESs adjacent to the dorsal or convex surface of spermatid heads and regions of Sertoli cell lobules known to contain distal parts of TBCs (Fig. 2a). Staining associated with the dorsal curvature of spermatid heads was intense while that associated with tubulobulbar regions was weaker and more diffuse. Staining with fluorescent phallotoxins for filamentous actin to highlight TBCs and ESs in this material was not successful because 
glutaraldehyde is used in the fixation protocol. Controls for staining were all negative (Fig. 2b-d).

\section{The early endosomal marker RAB5A is distinctly localized at the ends of TBCs}

When epithelial fragments containing late spermatids and associated Sertoli cell apical lobules were labeled both for filamentous actin and for RAB5A, staining for RAB5A occurred only at the ends of tubular regions of TBCs outlined by actin labeling (Fig. 3a-d). Small segments of actin staining also appeared to occur distal to the RAB5A-stained regions. In material fixed and processed for antigen retrieval, staining at the ends of TBCs was dramatically evident (Fig. 3e). The RAB5A staining occurred in regions similar to those reported above for nectin 2 and integrin, $\beta 1$. Controls for RAB5A staining were all negative (Fig. $3 \mathrm{f}-\mathrm{h}$ ), and the antibody reacted with a major band of the appropriate molecular weight $(24 \mathrm{kDa})$ for RAB5A on immunoblots of testis and seminiferous epithelial lysates (Fig. 3i). RAB5A staining on blots of whole testis and seminiferous epithelium was similar, indicating that tissues other than the epithelium also contain significant levels of RAB5A.

\section{The 'long-loop' recycling endosomal marker RAB11A is localized to newly forming junctions associated with early spermatids}

Staining with the immunological probe for RAB11A was dramatically evident in epithelial fragments adjacent to spermatids that were in the early stages of elongation
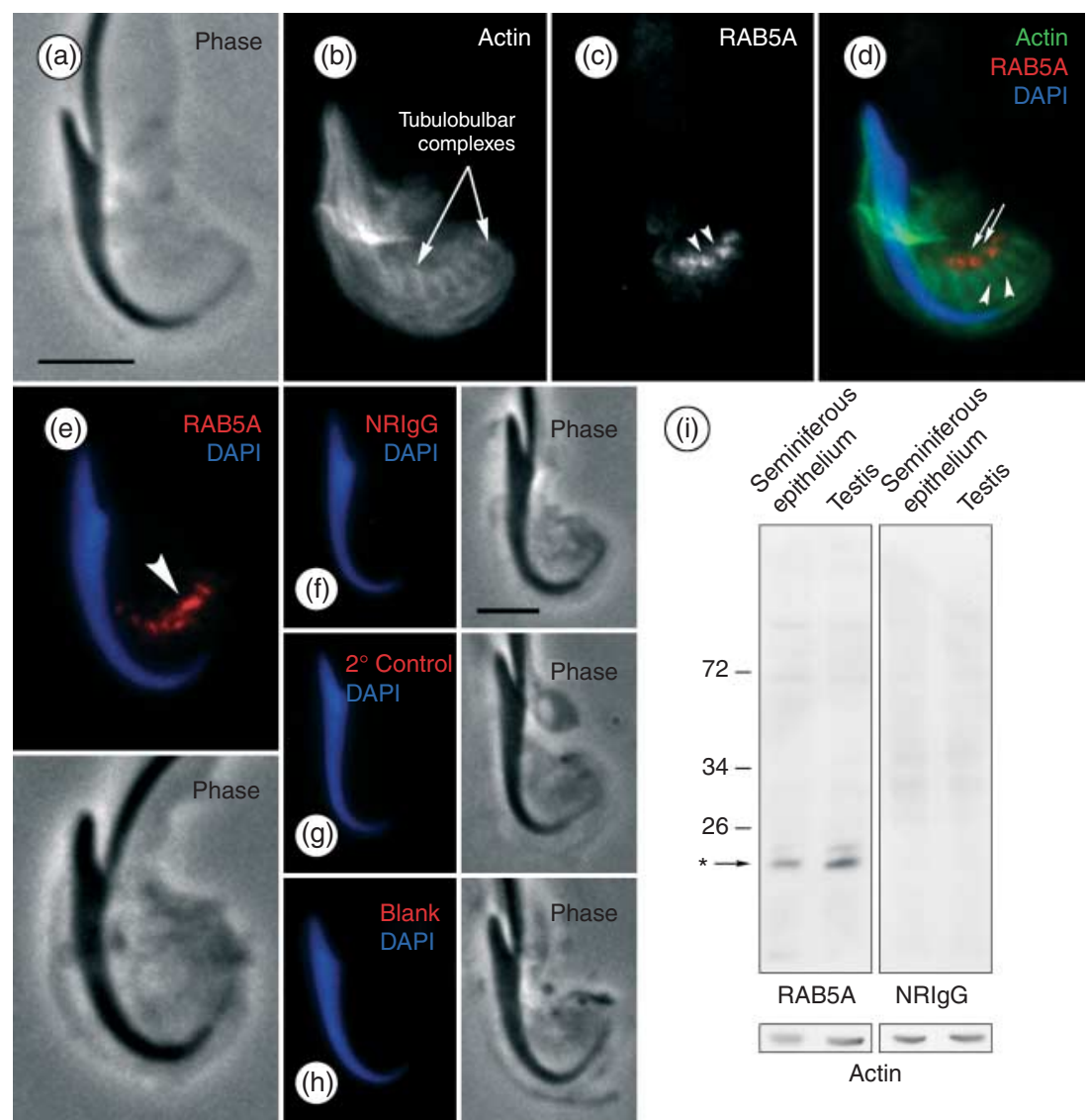

Figure 3 Phase and fluorescence images of late spermatids and associated Sertoli lobes mechanically dissociated from perfusion-fixed rat seminiferous epithelium and labeled for RAB5A. The epithelial fragment containing the spermatid shown in panels (a)-(d) was processed using our conventional immunofluorescence protocol. TBCs are clearly outlined by the actin labeling in (b). RAB5A labels regions of the complexes near their ends (arrowheads in (c)). These regions may correspond to the bulbar regions of the complexes because they occur precisely at the ends of the actinrelated proximal tubular regions (arrowheads in (d)). There is also some actin staining distal to the RAB5A-positive sites (arrows in (d)) in regions that may correspond to the short distal tubular regions of the complexes. (e) RAB5A staining in an epithelial fragment processed through the antigen retrieval protocol described in the text. The RAB5A antibody clearly labels TBCs near their ends (arrowhead). A similar pattern is absent in fragments treated with $(\mathrm{f})$ normal rabbit IgG (NRIgG) at the same concentration as that of primary antibody, (g) buffer instead of the primary antibody, and (h) buffer instead of both primary and the secondary antibodies. Bars $=5 \mu \mathrm{m}$. Panel (i) is a western blot of rat testis and seminiferous epithelium labeled for RAB5A. The RAB5A antibody reacts strongly with a major band $(*)$ of the appropriate molecular weight (24 kDa) for RAB5A on blots both of seminiferous epithelium and testis. Blots were re-probed for actin to control for loading (shown as the lower panel in the figure). DAPI, 4',6-diamidino-2-phenylindole; IgG, immunoglobulin G; RAB5A, RAB5A, member RAS oncogene family; TBC, tubulobulbar complex. 
(Fig. 4). Here, the labeling pattern was vesicular in appearance with the vesicles closely related to ESs indicated by actin staining. In early spermatids that were beginning to polarize (step 8 spermatids), RAB11Apositive puncta were often around the margins of the developing ESs (Fig. 4a), whereas somewhat later (step 9/
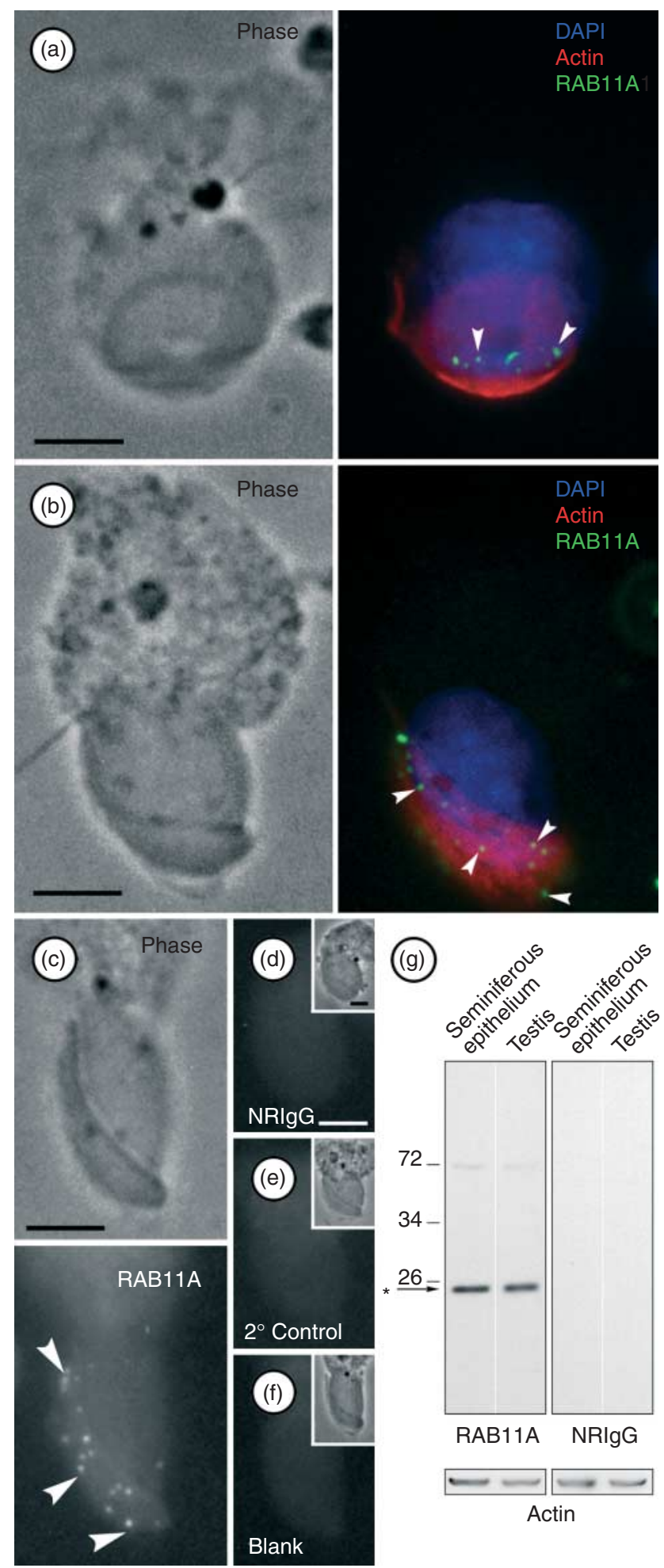

10) the vesicular staining was more widespread over the actin-positive junction (Fig. $4 \mathrm{~b}$ and c). Similar staining patterns were not observed in epithelial fragments treated with normal rabbit IgG at the same concentration as with primary antibody (Fig. 4d), when the primary antibody was replaced with buffer alone (Fig. 4e), or when both primary and secondary antibodies were replaced with buffer alone (Fig. 4f). On immunoblots of whole testis and isolated seminiferous epithelium, the antibody reacted with a single major band at the appropriate molecular weight $(22 \mathrm{kDa})$ for RAB11A (Fig. 4g), and like RAB5A, the protein did not appear enriched in seminiferous epithelium.

\section{RAB11A is co-distributed with nectin 2 at newly forming junctions}

To determine whether the junction molecules were co-distributed with RAB11A, we double labeled epithelial fragments with probes for RAB11A and nectin 2 . Significantly, no RAB11A staining occurred in Sertoli cell lobules associated with late spermatids, either at PVRL2positive ESs or at PVRL2-positive vesicles at the ends of TBCs (Fig. 5a). However, puncta clearly double labeled for PVRL2 and RAB11A were associated with regions known to contain ESs attached to early spermatids (Figs $5 \mathrm{~b}$ and $6 \mathrm{a}$ and $b$ ). When sections containing step 11 spermatids were labeled both for nectin 2 and RAB11A and then analyzed using ImageJ (National Institutes of Health, Bethesda, MD, USA) with a co-localization plug-in (Abramoff et al. 2004), the probes were co-localized in vesicular structures associated with apical junctions (Fig. 7).

When additional sections were analyzed with ImageJ (National Institutes of Health), it was confirmed that co-localization of PVRL2 and RAB11A was stage specific. There was little co-localization at apical junction sites in stages V and VII (Fig. 8a and b), whereas in stages containing early elongating spermatids where apical adhesion junctions (i.e. ESs) were newly forming

Figure 4 Paired phase and fluorescence micrographs of early spermatids labeled for actin, RAB11A, and DNA are shown in panels (a) and (b), while controls for RAB11A labeling are shown in panels (c)-(g). The spermatids in (a) and (b) are approximately at steps 8 and 9-10 of spermiogenesis respectively. Positive labeling for RAB11A occurs in a vesicular-like pattern (arrowheads) around the edge of the developing ectoplasmic specialization indicated by the actin labeling in (a). In panel (b), the positive vesicles (arrowheads) are more widely distributed over the developing junction. Bar $=5 \mu \mathrm{m}$. In panel (c), RAB11A-positive staining occurs in a punctate or vesicular pattern (arrowheads) adjacent to the early spermatid. Similar patterns are not seen in fragments incubated with (d) normal rabbit lgG (NRIgG) instead of primary antibody, and in secondary antibody controls in which (e) the primary antibody is replaced with buffer alone, or ( $\mathrm{f}$ ) both primary and secondary antibodies are replaced with buffer alone. (g) The RAB11A antibody reacts strongly with a single band $(*)$ on Western blots of seminiferous epithelium and testis, which is not present on similar blots treated with NRIgG alone. Blots were re-probed for actin (lower panel in (g)) to control for loading. Bars $=5 \mu \mathrm{m}$. 

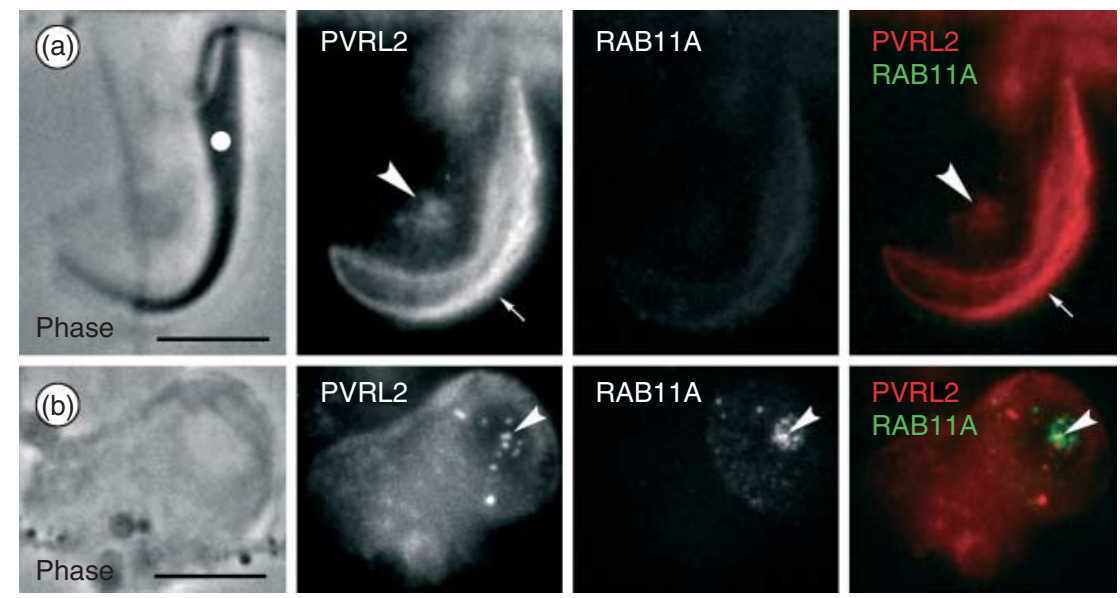

Figure 5 Phase and fluorescence micrographs of spermatids, and associated Sertoli cell regions, double labeled with antibodies to nectin 2 and RAB11A. (a) In association with late spermatids, the PVRL2 probe intensely labels ESs (arrow) associated with the spermatid head and also labels material in regions known to contain the ends of TBCs (arrowhead). RAB11A staining is negative in association with late spermatids. (b) In association with early elongate spermatids, nectin 2- and RAB11A-positive vesicles occur in Sertoli cell regions adjacent to the acrosome where adhesion junctions are being assembled. Some of these vesicles appear labeled for both probes. Note that some of the vesicles are nectin 2 positive and RAB11A negative. These vesicles may contain newly synthesized protein. Bars $=5 \mu \mathrm{m}$.

(Fig. 8c; stage IX/X), there was significantly more co-localization. Interestingly, co-localization at basal junctions between Sertoli cells was also more apparent (arrowheads in Fig. 8c) in stages containing early elongated spermatids. Here, basal junctions are turning over to allow the translocation of spermatocytes from basal to adluminal compartments of the epithelium.

\section{Discussion}

In this study, we show that both PVRL2 and $\beta 1$-integrin are present at the ends of TBCs in rat seminiferous epithelium and that similar regions are also associated with the early endosomal marker RAB5A. Significantly, we demonstrate that nectin 2 and the long recycling endosome marker RAB11A co-distribute at vesicles associated with ESs forming with early spermatids.

TBCs are tubular evaginations either of late spermatids or of Sertoli cells, which project into corresponding invaginations of adjacent Sertoli cells (Russell \& Clermont 1976, Russell 1979b). They form only at intercellular junctions. Although the structures occur at basal junctions between adjacent Sertoli cells, they have been best studied at apical sites of adhesion to late spermatids. Here, organized clusters of TBCs form, while at the same locations, ESs disassemble.

ESs are unique adhesion junctions in the seminiferous epithelium that are characterized in Sertoli cells by a layer of hexagonally packed actin filaments situated between the plasma membrane and a cistern of endoplasmic reticulum (Vogl et al. 2008). Unlike actinrelated adherens junctions in other epithelia that are cadherin based, ESs are mainly nectin and integrin based (Mulholland et al. 2001, Young et al. 2009b). At sites of Sertoli cell attachment to spermatids, nectin 2
(Ozaki-Kuroda et al. 2002) and integrin, a6ß1 (Palombi et al. 1992, Salanova et al. 1995) occur in the Sertoli cell membrane and their ligands on the spermatid are PVRL3 (Ozaki-Kuroda et al. 2002) and $\gamma 3$ laminin (Siu \& Cheng 2004) respectively.

We and others have presented data consistent with the conclusion that TBCs are subcellular machines that internalize intercellular junctions during junction remodeling in the seminiferous epithelium. At apical sites of Sertoli cell attachment to spermatids, the disassembly of adhesion junctions (i.e. ESs) occurs before sperm release and TBCs develop in areas previously occupied by ESs (Russell \& Clermont 1976, Guttman et al. 2004). The failure of apical TBCs to develop both in estradiol-treated rats (D'Souza et al. 2009) and in amphiphysin knockout animals (Kusumi et al. 2007) has been associated with spermiation failure. Moreover, spermiation failure is one of the first phenotypes observed in men undergoing hormonal methods of contraception (McLachlan et al. 2002). Vesicles associated with TBCs label positively for the lysosomal/endosomal markers acid phosphatase (ACP2; Russell 1979a, 1979b), lysosomal-associated membrane protein 1 (LAMP1), and sulphated glycoprotein 1/cathepsin (SGP1) (Guttman et al. 2004). In mice, PVRL2 and PVRL3 are concentrated in vesicles at the ends of TBCs (Guttman et al. 2004, Young et al. 2009b) and these vesicles are associated with EEA1 (Young et al. 2009b). In rats, $\alpha 6$-integrin (Young et al. 2009b), $\beta 1$-integrin (this paper), and PVRL2 (this paper) are concentrated in vesicles near the ends of TBCs. Significantly, the endosomal marker RAB5A clearly labels the ends of TBCs (this paper) in areas that appear proximal to those labeled by EEA1 in the mouse. Generally, in cells, RAB5A acts upstream of EEA1 in the endocytosis pathway (Christoforidis et al. 1999). 

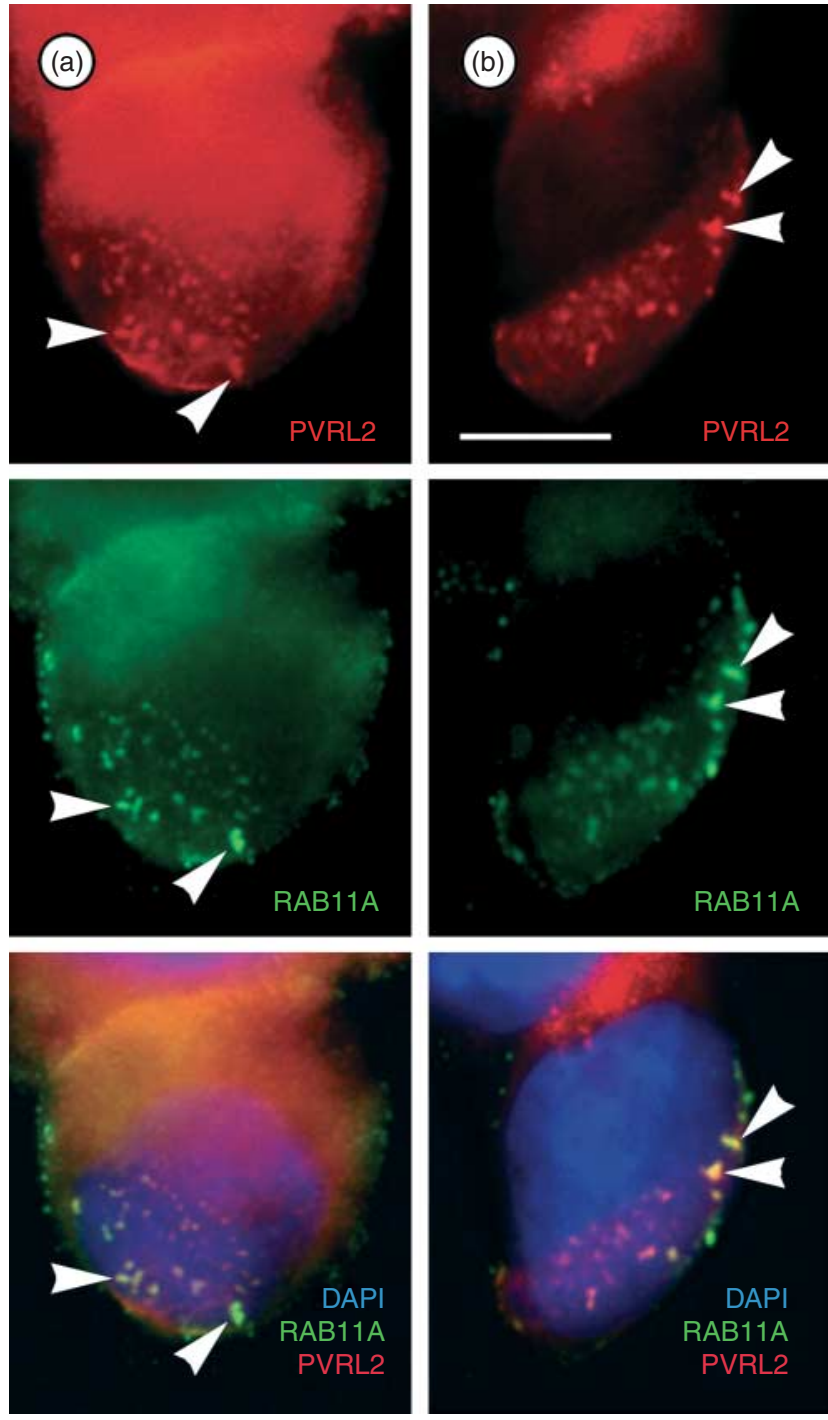

Figure 6 Immunofluorescence micrographs of early spermatids at (a) step 8 and (b) step 9 of spermiogenesis double labeled for nectin 2 and RAB11A. The cells are also stained with DAPI to highlight the nuclei. Both RAB11A and nectin 2 clearly label the same vesicular or punctate structures (arrowheads) in Sertoli cell regions overlying the acrosome regions of early spermatids. Also note that some of the vesicles that are RAB11A-positive do not label for nectin 2 . These vesicles may contain proteins other than nectin 2 that are also being recycled. Bars $=5 \mu \mathrm{m}$.

We suspect that the RAB5A-positive areas of TBCs may be the bulbar regions because they do not label for actin and are situated precisely at the ends of the strongly actinlabeled tubular regions. The concentration of adhesion molecules at the ends of TBCs and the presence of early endosomal markers in similar locations strongly support the argument that TBCs internalize intercellular junctions.

The fates of junction proteins internalized by TBCs have not been studied; however, by analogy with other systems, the proteins should be degraded and/or recycled. The appearance of what appear to be late endosomes/lysosomes in vesicular clusters associated with the ends of TBCs indicates that at least some of this material is degraded (Russell 1979a, Guttman et al. 2004). Also, nectin 3 in spermatid plasma membranes that is internalized by the Sertoli cell would presumably be degraded because this protein is not expressed by Sertoli cells. The situation may be very different for nectin 2 and $\alpha 6 \beta 1$-integrin. Both are expressed by Sertoli cells and are concentrated at ESs. Significantly, massive amounts of each presumably are internalized during sperm release at the apex of the epithelium while at the same time being added to the plasma membrane somewhat deeper in the epithelium in association with newly forming junctions with the next generation of spermatids. $\mathrm{RAB} 11 \mathrm{~A}$ regulates membrane trafficking into and out of the recycling endosome (Tarbutton et al. 2005). It has been implicated in the recycling of membrane components and transcytosis of molecules in polarized epithelia (Casanova et al. 1999, Xu et al. 2011). Our observation that nectin 2 positive vesicles that also label for the recycling endosomal marker RAB11A occur at newly forming ESs with early spermatids is the first indication that junction proteins internalized at TBCs may be recycled to newly forming junctions elsewhere in the epithelium (Fig. 9).

Because TBCs also occur in association with basal junction complexes between Sertoli cells at the sites of the 'blood-testis' barrier, we suspect that junctions are internalized by TBCs during basal junction turnover when spermatocytes translocate from basal to adluminal compartments of the epithelium; however, except for a report of tight and gap junctions being visible in electron micrographs of TBCs (Russell 1979b), evidence for this is lacking. During translocation, junctions disassemble above spermatocytes and new junctions assemble below (Russell 1977; Fig. 9). There is evidence from cultured Sertoli cells that junction proteins are endocytosed and recycled back to intercellular junctions (Yan \& Cheng 2005); however, it is not known whether this process involves TBCs or how these results translate into the intact seminiferous epithelium. One can speculate that recycling of junction proteins from TBCs may occur at basal junctions in a fashion similar to what occurs at apical sites. Our observation that nectin 2 and RAB11A are significantly more co-distributed at basal junctions at the stages of spermatogenesis where basal junctions are turning over to allow the next generation of spermatocytes through basal junction complexes is consistent with this argument. If recycling from basal TBCs does occur, it would be interesting to know whether adhesion proteins from apical and basal sites enter the same recycling compartments or are segregated. It also raises interesting questions about how the different junction proteins (adhesion, gap, and tight), which are presumably internalized together by TBCs at basal sites, are each trafficked through membrane recycling pathways.

Another issue yet to be clarified is whether junction proteins are differentially segregated by TBCs themselves during internalization. For example, nectins 2 and 3 

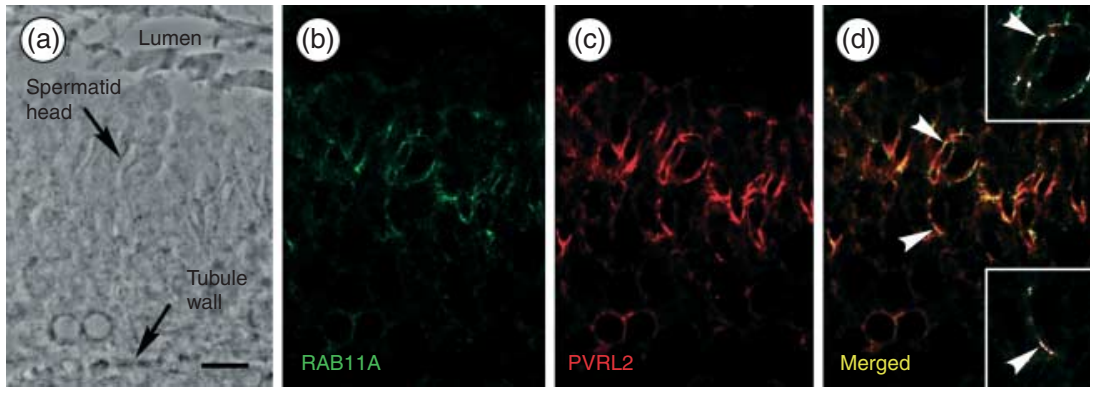

Figure 7 (a) A cryosection of a seminiferous tubule at stage XI containing step 11 spermatids and labeled both for (b) RAB11A and (c) for nectin 2 . The merged image is shown in panel $(d)$. The image was analyzed using Image (National Institutes of Health, Bethesda, MD, USA) and regions of colocalization are shown in white in the insets in (d) (arrowheads in (d) indicate the regions enlarged in the insets). The labeling with both probes is in regions known to contain $\mathrm{ESs}$. Bar $=20 \mu \mathrm{m}$.

might be entirely removed from apical junctions by TBCs, whereas $\alpha 6 \beta 1$ may be only partially removed. This would account for the presence of an integrin-based disengagement complex (Beardsley \& O'Donnell 2003, Beardsley et al. 2006) remaining as the last area of contact between Sertoli cells and spermatids after ESs and TBCs have disappeared.

In this paper, we demonstrate that the ends of TBCs at apical sites of attachment between Sertoli cells and late spermatids in rat are associated with the early endosomal marker RAB5A. Moreover, ITGB1 and PVRL2 also are concentrated at the ends of TBCs. Together, these data are consistent with the hypothesis that TBCs internalize apical junctions in the seminiferous epithelium. Our results also demonstrate that RAB11A and nectin 2 are co-localized at newly forming junctions with the next generation of spermatids and raise the interesting possibility that some of the junction proteins internalized during spermiation may be recycled to junctions forming deeper in the epithelium.

\section{Materials and Methods}

\section{Animals}

Animals used in this study were reproductively active male Sprague Dawley rats. They were obtained from Charles River and Harlan Animal Research Laboratories. The rats were maintained according to the guidelines established by the Canadian Council on Animal Care. All experiments were performed at least in duplicate using tissue from different animals.

\section{Reagents}

Unless otherwise indicated, all reagents used in the studies were obtained from Sigma-Aldrich. Paraformaldehyde was obtained from Fisher Scientific Limited (Ottawa, ON, Canada). The secondary antibodies and phallotoxins conjugated to Alexa fluorochromes were obtained from Invitrogen and those conjugated to HRP were purchased from Jackson ImmunoResearch Laboratories, Inc. (Westgrove, PA, USA).

\section{Antibodies}

Primary antibodies were obtained from the following sources and used at the indicated working concentrations for immunostaining: rabbit anti-PVRL2- $\delta$ (generated in the laboratory of Dr Takai), $1.0 \mu \mathrm{g} / \mathrm{ml}$; goat polyclonal anti-RAB11A (sc-6565; Santa Cruz Biotechnologies, Santa Cruz, CA, USA), $5.0 \mu \mathrm{g} / \mathrm{ml}$; rabbit polyclonal Rab5 (ab18211; Abcam plc, Cambridge, UK), $10.0 \mu \mathrm{g} / \mathrm{ml}$; rabbit anti- $\beta 1$-integrin ( $\mathrm{AB}$ 1952; Millipore, Billerica, MA, USA), $2.0 \mu \mathrm{g} / \mathrm{ml}$; and mouse anti- $\beta$-actin (A1978; Sigma-Aldrich), $5.0 \mu \mathrm{g} / \mathrm{ml}$.

\section{Immunofluorescence}

\section{Tissue preparation}

Animals were placed under deep anesthesia by halothane inhalation, the testes were removed, and then the animals were killed. Spermatic arteries were cannulated with 26-gauge needles and gravity perfused with warm PBS $(150 \mathrm{mM} \mathrm{NaCl}$, $5 \mathrm{mM} \mathrm{KCl}, 0.8 \mathrm{mM} \mathrm{KH}_{2} \mathrm{PO}_{4}$, and $3.2 \mathrm{mM} \mathrm{Na}_{2} \mathrm{HPO}_{4}, \mathrm{pH} 7.3$ at $\left.33{ }^{\circ} \mathrm{C}\right)$ for 2 min to clear blood and then with warm fixative $(3 \%$ paraformaldehyde, $150 \mathrm{mM} \mathrm{NaCl}, 5 \mathrm{mM} \mathrm{KCl}, 0.8 \mathrm{mM}$ $\mathrm{KH}_{2} \mathrm{PO}_{4}$, and $3.2 \mathrm{mM} \mathrm{NaHPO}$, $\mathrm{pH} 7.3$ at $33^{\circ} \mathrm{C}$ ) for $30 \mathrm{~min}$. Fixation was followed by a 30-min wash with PBS.

\section{Fragmented material}

Fixed testes were decapsulated in PBS and the seminiferous tubules were mechanically separated into small pieces $(\sim 1-$ $2 \mathrm{~mm}$ cubes) using scalpels and then transferred into a $50 \mathrm{ml}$ Falcon tube. The material was gently aspirated through an 18-gauge needle and then through a 21-gauge needle to fragment the epithelium. Larger material was allowed to sediment for $10 \mathrm{~min}$ and the supernatant was collected. Using low-speed centrifugation, epithelial fragments were precipitated and then re-suspended in fresh PBS. This material was then added to poly-L-lysine-coated slides. The slides were allowed to incubate in a humidity chamber for $5 \mathrm{~min}$ in order for the tissue to adhere to the slide. Excess fluid was removed and the slides were placed in cold acetone $\left(-20^{\circ} \mathrm{C}\right)$ for $5 \mathrm{~min}$ and air-dried.

\section{Fixed frozen sections}

Fixed testes were placed on aluminum stubs with a small amount of optimal cutting temperature compound between the stub and the tissue (Sakura Finetek USA, Inc., Torrance, CA, USA) and frozen using liquid nitrogen. Frozen sections ( $5 \mu \mathrm{m})$ were cut, collected on poly-L-lysine-coated slides, and immediately plunged into cold acetone $\left(-20^{\circ} \mathrm{C}\right)$ for $5 \mathrm{~min}$ and air-dried.

\section{Immunostaining}

Slides with the attached tissue fragments or cryosections were treated with $5 \%$ normal goat serum (NGS) in TPBS-BSA (PBS containing $0.05 \%$ Tween 20 and $0.1 \%$ BSA) (TPBS-BSA) for 

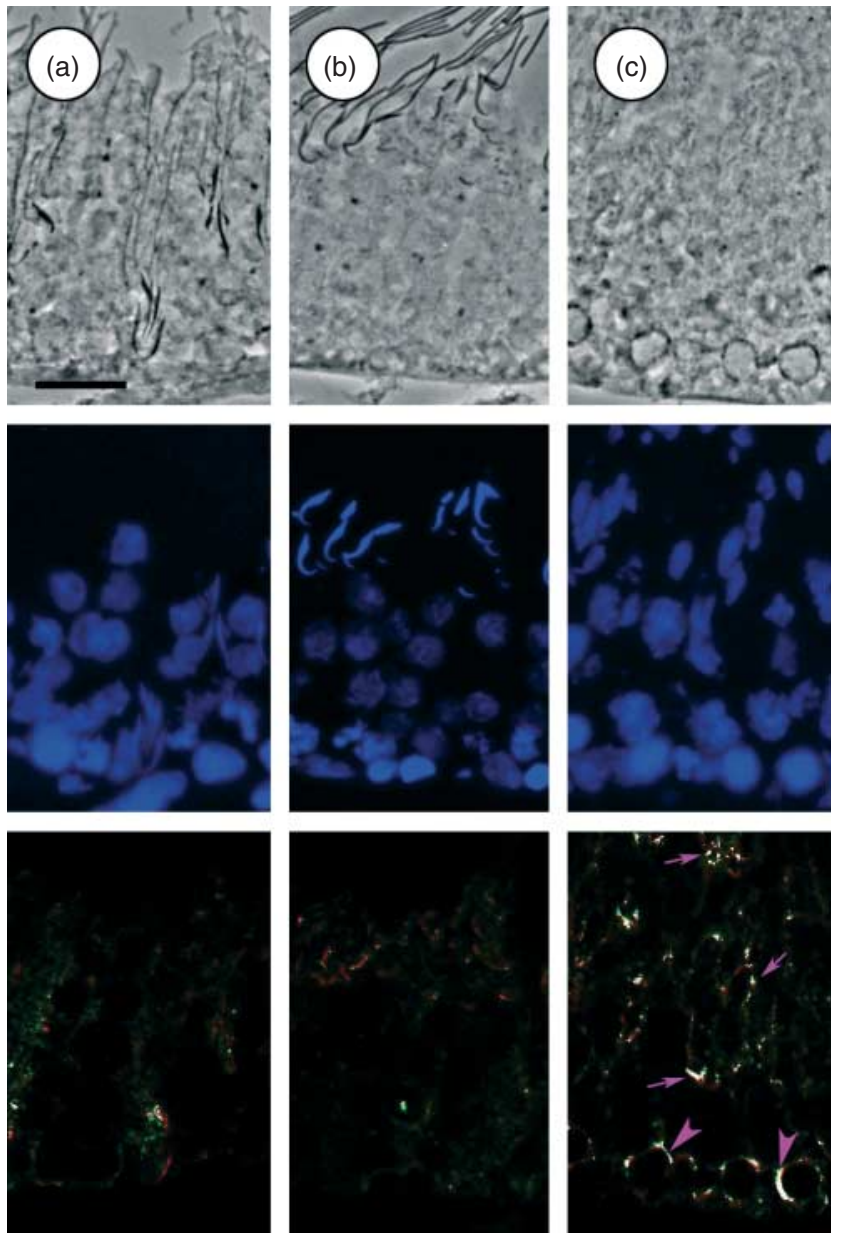

Figure 8 Cryosections of seminiferous tubules at stages (a) V, (b) VII, and (c) IX of spermatogenesis that have been stained for nectin 2 (red channel) and RAB11A (green channel). The images were analyzed using ImageJ (National Institutes of Health, Bethesda, MD, USA) to highlight areas of co-localization. The upper panels are phase, the middle panels are stained with DAPI to show the nuclei, and the lower panels illustrate areas where nectin 2 and RAB11A are co-localized (shown in white). Note that co-localization (pink arrows) is most apparent at stage IX where new adhesion junctions are forming with the next generation of early elongating spermatids. At the same stage, co-localization in the basal regions of the epithelium (pink arrowheads), where junctions are beginning to turn over to allow the translocation of spermatocytes from basal to adluminal compartments of the epithelium, is also more apparent than at the earlier stages. Bar $=50 \mu \mathrm{m}$. PVRL2, nectin 2; RAB11A, RAB11A, member RAS oncogene family; DAPI, 4',6-diamidino-2-phenylindol.

$20 \mathrm{~min}$ at room temperature. Primary antibodies were added to the experimental slides, made up in TPBS-BSA with $1 \%$ NGS, and incubated overnight at $4{ }^{\circ} \mathrm{C}$ in a humidity chamber. The material was washed extensively with TPBS-BSA (wash buffer) and incubated for $1 \mathrm{~h}$ at $37^{\circ} \mathrm{C}$ with secondary antibody conjugated to a fluorochrome. The slides were again washed and then coverslips were mounted using VECTASHIELD Mounting Medium (VECTOR Laboratories, Inc. Burlingame, CA, USA). The tissue was visualized using a Zeiss Axiophot microscope fitted with appropriate filter sets for detecting fluorescence and with the appropriate optics for phase contrast microscopy. Antibodies to $\beta 1$-integrin and RAB5A could not be co-localized because they were generated in the same host animal.

Controls for immunofluorescence included 1) primary antibodies replaced with normal $\lg G$ from the host animal species at identical concentrations to the primary antibody (specificity control); 2) primary antibody replaced with buffer alone (secondary antibody control); and 3) both primary and secondary antibodies replaced with buffer alone (control for autofluorescence - blank).

\section{Actin staining}

Filamentous actin was labeled using ALEXA 488 or ALEXA 568 phalloidin (Molecular Probes, Burlington, ON, Canada). The stain was prepared as specified by the manufacturer in TPBSBSA. For each slide, $12.5 \mu \mathrm{l}$ stock ALEXA phalloidin (in methanol) was taken in a glass test tube, evaporated to dryness,

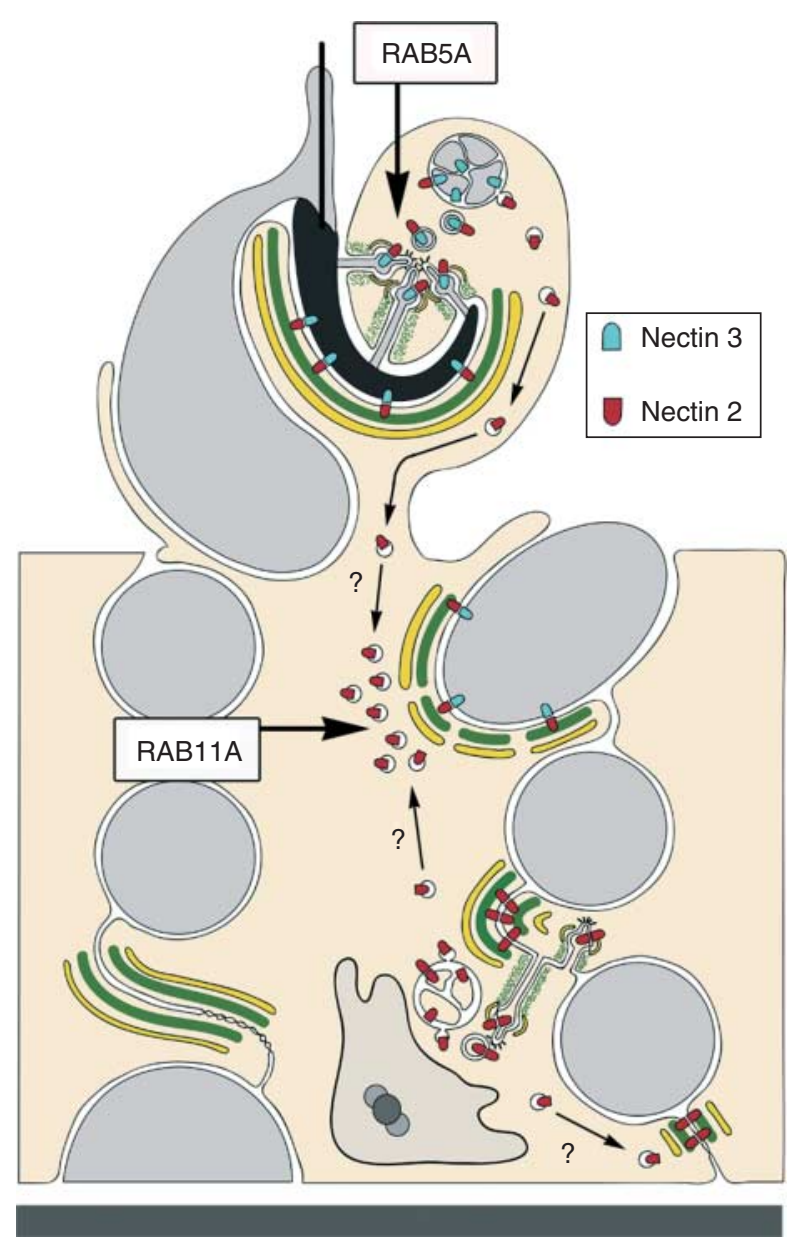

Figure 9 Model of junction turnover and recycling in the seminiferous epithelium of the rat, partly based on data presented in this paper. Nectin 2 is internalized into the Sertoli cell at apical TBCs where the internalization machinery is associated with RAB5A. Some of the adhesion protein enters recycling compartments in the cell and is targeted to newly forming adhesion junctions with early spermatids deeper in the epithelium. RAB11A is associated with nectin 2 containing compartments associated with these early spermatids. We speculate that a similar process may occur at basal junction sites. 
and then the material was rehydrated with $25 \mu \mathrm{I}$ TPBS-BSA and added to the slide.

\section{Antigen retrieval}

Because the $\beta 1$-integrin antibodies did not react on tissue processed using standard techniques for immunofluorescence, we explored different fixation protocols and the use of antigen retrieval methods to recover antigenicity. Other studies have used the same antibody to label paraffin sections using antigen retrieval (Beardsley \& O'Donnell 2003). For all $\beta 1$-integrin experiments, the tissue was processed as described above except that the fixative also contained $0.1 \%$ glutaraldehyde and slides were not processed through acetone or allowed to air-dry. Instead, slides were immersed in hot $\left(95-100{ }^{\circ} \mathrm{C}\right)$ sodium citrate buffer $(10 \mathrm{mM}$ sodium citrate, $0.05 \%$ Tween 20 , $\mathrm{pH}$ 6.0) immediately after letting epithelial fragments settle onto the slides and removing excess fluid. After $5 \mathrm{~min}$, the slides were removed from the buffer and immunostained. We also used this protocol for some of the RAB5A experiments and found that staining was improved, possibly because the fixative contained a small amount of glutaraldehyde.

\section{Western blotting}

Western blot analyses were performed using whole testis and seminiferous epithelium lysates. Testes were removed from animals under deep anesthesia and decapsulated. Seminiferous epithelium was isolated as described elsewhere (Vaid et al. 2007). Both the seminiferous epithelium and whole testis were homogenized in an RIPA lysis buffer $(150 \mathrm{mM} \mathrm{NaCl}, 50 \mathrm{mM}$ Tris, pH 7.4, 5 mM EDTA, 1\% Nonidet P-40, 1\% deoxycholic acid, and $0.1 \%$ SDS) and flash frozen in liquid nitrogen for storage at $-80{ }^{\circ} \mathrm{C}$. When ready for use, lysate was thawed at room temperature, added to the sample buffer, and boiled for 10 min before being loaded into wells of 1 -mm-thick $12 \%$ acrylamide gels for SDS-PAGE at equal concentrations as determined by protein assay and run under reducing conditions at $70 \mathrm{~V}$ for $3 \mathrm{~h}$. Proteins were transferred onto Immobilon-P transfer membrane (Millipore) and then washed for $5 \mathrm{~min}$ in $500 \mathrm{mM}$ Tris, $\mathrm{pH} 7.5,150 \mathrm{mM} \mathrm{NaCl}$, and $0.1 \%$ (TBST). The blots were blocked overnight at $4{ }^{\circ} \mathrm{C}$ using $4 \%$ non-fat milk (sc-2325; Santa Cruz Biotechnologies) to decrease nonspecific binding of antibodies. Blots were washed three times for 10 min each and then incubated at room temperature with relevant primary antibodies and controls at appropriate concentrations. After $60 \mathrm{~min}$, the blots were washed with TBST followed by a 60-min room temperature wash with a corresponding secondary antibody conjugated to HRP at appropriate concentrations. After washing with TBST, the blots were treated with ECL (Amersham) and bands visualized with Bioflex Econofilm (InterScience, Markham, ON, Canada). Controls consisted of replacing the primary antibodies with normal $\operatorname{lgG}$ at the same concentration as the primary antibody.

To control for protein loading in the RAB5A and RAB11A experiments, the membranes were stripped in methanol for $1 \mathrm{~h}$ at room temperature, washed with TBST, and blocked overnight at $4{ }^{\circ} \mathrm{C}$ in $4 \%$ milk. The blocking step was followed by a TBST wash, a 1-h room temperature incubation with $\beta$-actin, and another TBST wash. The membrane was then incubated in the corresponding secondary antibody for $1 \mathrm{~h}$, washed once more with TBST, treated with ECL, and exposed.

\section{Declaration of interest}

The authors declare that there is no conflict of interest that could be perceived as prejudicing the impartiality of the research reported.

\section{Funding}

This work was supported by the Natural Sciences and Engineering Research Council of Canada grant RGPIN 155397-08 to A W Vogl.

\section{Acknowledgements}

The authors thank Maxence LeVasseur for his help with imaging and analysis using ImageJ.

\section{References}

Abramoff MD, Magalhaes PJ \& Ram SJ 2004 Image processing with ImageJ. Biophotonics International 11 36-42.

Akhtar N \& Hotchin NA 2001 RAC1 regulates adherens junctions through endocytosis of E-cadherin. Molecular Biology of the Cell 12 847-862.

Baum B \& Georgiou M 2011 Dynamics of adherens junctions in epithelial establishment, maintenance, and remodeling. Journal of Cell Biology 192 907-917. (doi:10.1083/jcb.201009141)

Beardsley A \& O'Donnell L 2003 Characterization of normal spermiation and spermiation failure induced by hormone suppression in adult rats. Biology of Reproduction 68 1299-1307. (doi:10.1095/biolreprod.102. 009811)

Beardsley A, Robertson DM \& O'Donnell L 2006 A complex containing alpha6beta1-integrin and phosphorylated focal adhesion kinase between Sertoli cells and elongated spermatids during spermatid release from the seminiferous epithelium. Journal of Endocrinology 190 759-770. (doi:10.1677/joe.1.06867)

Berthoud VM, Minogue PI, Laing JG \& Beyer EC 2004 Pathways for degradation of connexins and gap junctions. Cardiovascular Research 62 256-267. (doi:10.1016/j.cardiores.2003.12.021)

Casanova JE, Wang X, Kumar R, Bhartur SG, Navarre J, Woodrum JE, Altschuler Y, Ray GS \& Goldenring JR 1999 Association of Rab25 and RAB11Aa with the apical recycling system of polarized Madin-Darby canine kidney cells. Molecular Biology of the Cell 10 47-61.

Caswell PT \& Norman JC 2006 Integrin trafficking and the control of cell migration. Traffic 7 14-21. (doi:10.1111/j.1600-0854.2005.00362.x)

Chao WT \& Kunz J 2009 Focal adhesion disassembly requires clathrindependent endocytosis of integrins. FEBS Letters 583 1337-1343. (doi:10.1016/j.febslet.2009.03.037)

Christoforidis S, McBride HM, Burgoyne RD \& Zerial M 1999 The Rab5 effector EEA1 is a core component of endosome docking. Nature 397 621-625. (doi:10.1038/17618)

D'Souza R, Pathak S, Upadhyay R, Gaonkar R, D'Souza S, Sonawane S, Gill-Sharma M \& Balasinor NH 2009 Disruption of tubulobulbar complex by high intratesticular estrogens leading to failed spermiation. Endocrinology 150 1861-1869. (doi:10.1210/en.2008-1232)

Dym M \& Fawcett DW 1970 The blood-testis barrier in the rat and the physiological compartmentation of the seminiferous epithelium. Biology of Reproduction 3 308-326.

Gaietta G, Deerinck TJ, Adams SR, Bouwer J, Tour O, Laird DW, Sosinsky GE, Tsien RY \& Ellisman MH 2002 Multicolor and electron microscopic imaging of connexin trafficking. Science 296 503-507. (doi:10.1126/science.1068793) 
Guttman JA, Takai Y \& Vogl AW 2004 Evidence that tubulobulbar complexes in the seminiferous epithelium are involved with internalization of adhesion junctions. Biology of Reproduction 71 548-559. (doi:10.1095/biolreprod.104.028803)

Ivanov AI, Nusrat A \& Parkos CA 2004 Endocytosis of epithelial apical junctional proteins by a clathrin-mediated pathway into a unique storage compartment. Molecular Biology of the Cell 15 176-188. (doi:10.1091/ mbc.E03-05-0319)

Izumi G, Sakisaka T, Baba T, Tanaka S, Morimoto K \& Takai Y 2004 Endocytosis of E-cadherin regulated by Rac and Cdc42 small G proteins through IQGAP1 and actin filaments. Journal of Cell Biology 166 237-248. (doi:10.1083/jcb.200401078)

Kusumi N, Watanabe M, Yamada H, Li SA, Kashiwakura Y, Matsukawa T, Nagai A, Nasu Y, Kumon H \& Takei K 2007 Implication of amphiphysin 1 and dynamin 2 in tubulobulbar complex formation and spermatid release. Cell Structure and Function 32 101-113. (doi:10.1247/csf.07024)

Le TL, Yap AS \& Stow JL 1999 Recycling of E-cadherin: a potential mechanism for regulating cadherin dynamics. Journal of Cell Biology 146 219-232.

McLachlan RI, O'Donnell L, Stanton PG, Balourdos G, Frydenberg M, de Kretser DM \& Robertson DM 2002 Effects of testosterone plus medroxyprogesterone acetate on semen quality, reproductive hormones, and germ cell populations in normal young men. Journal of Clinical Endocrinology and Metabolism 87 546-556. (doi:10.1210/jc.87.2.546)

Mulholland DJ, Dedhar S \& Vogl AW 2001 Rat seminiferous epithelium contains a unique junction (ectoplasmic specialization) with signaling properties both of cell/cell and cell/matrix junctions. Biology of Reproduction 64 396-407. (doi:10.1095/biolreprod64.1.396)

Ozaki-Kuroda K, Nakanishi H, Ohta H, Tanaka H, Kurihara H, Mueller S, Irie K, Ikeda W, Sakai T, Wimmer E et al. 2002 Nectin couples cell-cell adhesion and the actin scaffold at heterotypic testicular junctions. Current Biology 12 1145-1150. (doi:10.1016/S0960-9822(02)00922-3)

Palombi F, Salanova M, Tarone G, Farini D \& Stefanini M 1992 Distribution of beta 1 integrin subunit in rat seminiferous epithelium. Biology of Reproduction 47 1173-1182. (doi:10.1095/biolreprod47.6.1173)

Paterson AD, Parton RG, Ferguson C, Stow JL \& Yap AS 2003 Characterization of E-cadherin endocytosis in isolated MCF-7 and chinese hamster ovary cells: the initial fate of unbound E-cadherin. Journal of Biological Chemistry 278 21050-21057. (doi:10.1074/jbc.M300082200)

Piehl M, Lehmann C, Gumpert A, Denizot JP, Segretain D \& Falk MM 2007 Internalization of large double-membrane intercellular vesicles by a clathrin-dependent endocytic process. Molecular Biology of the Cell 18 337-347. (doi:10.1091/mbc.E06-06-0487)

Russell L 1977 Movement of spermatocytes from the basal to the adluminal compartment of the rat testis. American Journal of Anatomy 148 313-328. (doi:10.1002/aja.1001480303)

Russell LD 1979a Further observations on tubulobulbar complexes formed by late spermatids and Sertoli cells in the rat testis. Anatomical Record 194 213-232. (doi:10.1002/ar.1091940204)

Russell LD $1979 \mathrm{~b}$ Observations on the inter-relationships of Sertoli cells at the level of the blood-testis barrier: evidence for formation and resorption of Sertoli-Sertoli tubulobulbar complexes during the spermatogenic cycle of the rat. American Journal of Anatomy 155 259-279. (doi:10.1002/aja.1001550208)

Russell LD 1979c Spermatid-Sertoli tubulobulbar complexes as devices for elimination of cytoplasm from the head region late spermatids of the rat. Anatomical Record 194 233-246. (doi:10.1002/ar.1091940205)

Russell L \& Clermont Y 1976 Anchoring device between Sertoli cells and late spermatids in rat seminiferous tubules. Anatomical Record 185 259-278. (doi:10.1002/ar.1091850302)
Salanova M, Stefanini M, De Curtis I \& Palombi F 1995 Integrin receptor alpha 6 beta 1 is localized at specific sites of cell-to-cell contact in rat seminiferous epithelium. Biology of Reproduction 52 79-87. (doi:10. 1095/biolreprod52.1.79)

Shen L \& Turner JR 2005 Actin depolymerization disrupts tight junctions via caveolae-mediated endocytosis. Molecular Biology of the Cell 16 3919-3936. (doi:10.1091/mbc.E04-12-1089)

Siu MK \& Cheng CY 2004 Interactions of proteases, protease inhibitors, and the beta1 integrin/laminin gamma3 protein complex in the regulation of ectoplasmic specialization dynamics in the rat testis. Biology of Reproduction 70 945-964. (doi:10.1095/biolreprod.103.023606)

Tarbutton E, Peden AA, Junutula JR \& Prekeris R 2005 Class I FIPs, RAB11Abinding proteins that regulate endocytic sorting and recycling. Methods in Enzymology 403 512-525. (doi:10.1016/S0076-6879(05)03045-4)

Troyanovsky RB, Sokolov EP \& Troyanovsky SM 2006 Endocytosis of cadherin from intracellular junctions is the driving force for cadherin adhesive dimer disassembly. Molecular Biology of the Cell 17 3484-3493. (doi:10.1091/mbc.E06-03-0190)

Utech M, Ivanov AI, Samarin SN, Bruewer M, Turner JR, Mrsny RJ, Parkos CA \& Nusrat A 2005 Mechanism of IFN-gamma-induced endocytosis of tight junction proteins: myosin II-dependent vacuolarization of the apical plasma membrane. Molecular Biology of the Cell 16 5040-5052. (doi:10.1091/mbc.E05-03-0193)

Vaid KS, Guttman JA, Babyak N, Deng W, McNiven MA, Mochizuki N, Finlay BB \& Vogl AW 2007 The role of dynamin 3 in the testis. Journal of Cellular Physiology 210 644-654. (doi:10.1002/jcp.20855)

Vogl AW, Vaid KS \& Guttman JA 2008 The Sertoli cell cytoskeleton. In Molecular Mechanisms in Spermatogenesis, Advances in Experimental Medicine and Biology, vol 636, pp 186-211. Ed. CY Cheng. Austin, TX: Springer Sciences + Buisness Media, LLC Landes Bioscience.

Wu M, Huang B, Graham M, Raimondi A, Heuser JE, Zhuang X \& De Camilli P 2010 Coupling between clathrin-dependent endocytic budding and F-BAR-dependent tubulation in a cell-free system. Nature Cell Biology 12 902-908. (doi:10.1038/ncb2094)

Xu S, Edman M, Kothawala MS, Sun G, Chiang L, Mircheff A, Zhu L, Okamoto C \& Hamm-Alvarez S 2011 A RAB11A-enriched subapical membrane compartment regulates a cytoskeleton-dependent transcytotic pathway in secretory epithelial cells of the lacrimal gland. Journal of Cell Science 124 3503-3514. (doi: 10.1242/jcs.088906)

Yan HH \& Cheng CY 2005 Blood-testis barrier dynamics are regulated by an engagement/disengagement mechanism between tight and adherens junctions via peripheral adaptors. PNAS 102 11722-11727. (doi:10.1073/pnas.0503855102)

Young JS, Guttman JA, Vaid KS, Shahinian H \& Vogl AW 2009a Cortactin (CTTN), N-WASP (WASL), and clathrin (CLTC) are present at podosomelike tubulobulbar complexes in the rat testis. Biology of Reproduction $\mathbf{8 0}$ 153-161. (doi:10.1095/biolreprod.108.070615)

Young JS, Guttman JA, Vaid KS \& Vogl AW $2009 b$ Tubulobulbar complexes are intercellular podosome-like structures that internalize intact intercellular junctions during epithelial remodeling events in the rat testis. Biology of Reproduction 80 162-174. (doi:10.1095/biolreprod. 108.070623)

Received 24 August 2011

First decision 5 October 2011

Revised manuscript received 1 December 2011

Accepted 12 December 2011 